\title{
DESEMPENHO DE METODOLOGIAS PARA ESTIMATIVA DO SALDO DE RADIAÇÃO A PARTIR DE IMAGENS MODIS
}

\section{FRANCINEIDE AMORIM COSTA SANTOS ${ }^{1}$,CARLOS ANTONIO COSTA DOS SANTOS ${ }^{1}$,BERNARDO BARBOSA DA SILVA ${ }^{2}$, ARGEMIRO LUCENA ARAÚJO ${ }^{3}$, JOHN ELTON DE BRITO LEITE CUNHA ${ }^{4}$}

\author{
${ }^{1}$ Universidade Federal de Campina Grande, Unidade Acadêmica de Ciências Atmosféricas, \\ Campina Grande, PB, Brasil \\ ${ }^{2}$ Universidade Federal de Pernambuco, Centro de Tecnologia, Departamento de Engenharia Civil, \\ Recife, PE, Brasil \\ ${ }^{3}$ Campina Geotech, Campina Grande, PB, Brasil \\ ${ }^{4}$ Universidade Federal de Campina Grande, Unidade Acadêmica de Tecnologia do Desenvolvimento, \\ Sumé, PB, Brasil
}

francyacs@hotmail.com,carlos.santos@ufcg.edu.br, bbdasilva.ufpe@gmail.com, argemirolucena@gmail.com,john.e.cunha@gmail.com

Recebido Outubro de 2013 - Aceito Dezembro de 2014

\begin{abstract}
RESUMO
O presente estudo objetivou avaliar o desempenho de diferentes metodologias utilizadas na estimativa do saldo de radiação instantâneo (Rn), que é a principal fonte de energia em diversos processos naturais que ocorrem na superfície do nosso planeta. A área de estudo pertence à Bacia Hidrográfica do Rio Mogi-Guaçu. Os dados de superfície que foram empregados na calibração e validação do Rn, foram obtidos de duas torres micrometeorológicas instaladas em áreas de cerrado e de cana-de-açúcar. Foram utilizadas imagens do sensor MODIS e as metodologias empregadas foram SEBAL, METRIC e o modelo proposto por Bisht et al. O Rn no cerrado se mostrou sempre maior que o da cana-de-açúcar evidenciando que, nessas áreas nativas, há, portanto, uma maior quantidade de energia a ser convertida em calor sensível e latente. As metodologias empregadas mostraram-se bastante satisfatórias, havendo um melhor desempenho da metodologia METRIC. O fato da metodologia de Bisht et al. não necessitar de dados de superfície mostra-se bastante útil, já que na falta de medições de superfície tem-se a opção prática e sem custo para o usuário de fazer as estimativas do saldo de radiação. Palavras chave: albedo; índice de vegetação; mudança climática; produtos MODIS
\end{abstract}

\begin{abstract}
PERFORMANCE OF METHODOLOGIES FOR ESTIMATING NET RADIATION FROM MODIS IMAGES

The present study intends to evaluate the performance of different methodologies used in estimating the instantaneous net radiation ( $\mathrm{Rn}$ ), which is the main energy source to various natural processes occurring on the surface of our planet. The study area belongs to the Mogi Guacu River Basin. The surface data used for the Rn calibration and validation are from two micrometeorological towers located in areas of cerrado and sugarcane. MODIS sensor images and the SEBAL, METRIC and Bisht et al. methodologies were compared. $\mathrm{Rn}$ in the cerrado area always showed higher values than that of sugarcane, indicating that, in those wild areas, there is therefore a greater amount of energy to transform into latent and sensible heat fluxes. The employed methodologies were quite satisfactory, with a best performance of the METRIC methodology. The fact that the methodology Bisht et al. does not require surface data appears to be quite useful, since in the absence of surface measurements it has been a frequent and no-cost option for the user to make estimates of net radiation.
\end{abstract}

Keywords: albedo; vegetation indexes; climate change; MODIS products 


\section{INTRODUÇÃO}

O saldo de radiação $(\mathrm{Rn})$ na superfície da Terra tem grande importância nos processos de evaporação, fotossíntese e aquecimentos do solo e do ar, e pode ser quantificado mediante a contabilidade entre os fluxos radiativos ascendentes e descendentes, incluindo ondas longas e curtas. A sua quantificação é fundamental para estudar as interações biosfera-atmosfera (Bisht e Bras, 2010). Dessa forma, é de grande importância investigar alterações que possam ser estabelecidas nas componentes do saldo de Radiação à superfície, especialmente no que diz respeito ao aumento de temperatura da superfície e albedo, que impactam diretamente a transferência de massa (água e $\mathrm{CO}_{2}$ ) para a atmosfera.

Em face da grande expansão demográfica, conjugada com a ausência de políticas públicas eficazes e pertinentes ao ordenamento do território e às questões ambientais, as áreas de vegetação nativa no Brasil vêm sendo substituídas ao longo de décadas por cultivos agrícolas. No estado de São Paulo, em particular, à época do descobrimento, a Mata Atlântica recobria aproximadamente $81 \%$ de sua área, sendo o restante ocupado principalmente pelo Cerrado e campos naturais (SMA, 2010). Atualmente, existe uma área remanescente de cobertura vegetal equivalente a $13,94 \%$ do território, dos quais cerca de 13\% correspondem ao domínio Mata Atlântica e apenas 1\% ao Cerrado. Fato que chama a atenção é a expansão da canade-açúcar, cuja área ocupada dobrou entre os anos agrícolas $1995 / 1996$ e 2007/2008. Na safra 2008/2009 ultrapassou os 4,5 milhões de hectares, totalizando quase dois terços do total das áreas de lavoura do Estado (SMA, 2010).

Estudos recentes que tratam da substituição do cerrado por cana-de-açúcar (Negrón-Juarez, 2004; Tatsch, 2006; Gomes, 2009) têm mostrado que ocorre redução no saldo de radiação e na evapotranspiração média anual, e aumento na temperatura do ar sobre o dossel da plantação, em comparação ao cerrado. Segundo experimentos numéricos reportados por NegrónJuarez (2004), a substituição da vegetação primitiva no Sudeste brasileiro pela cana-de-açúcar pode produzir mudanças no padrão da distribuição espacial de chuvas e temperatura (redução da precipitação anual em 5\%, aproximadamente, e aumento de até $0,6^{\circ} \mathrm{C}$ na temperatura do ar à superfície).

Existem muitas vantagens ao se fazer uso do sensoriamento remoto orbital com o propósito de identificar alterações no uso da terra e as alterações climáticas advindas de tais mudanças. Destaque-se a grande cobertura espacial, a facilidade de obtenção das imagens e a possibilidade de estimar alguns parâmetros que só seriam obtidos com instrumentos normalmente utilizados em estudos experimentais. Ademais, os resultados obtidos são representativos de áreas com características idênticas, no aspecto do porte, estado hídrico e condições atmosféricas. As técnicas baseadas em imagens de satélite, ao contrário, cobrem grandes extensões terrestres.

Uma questão de grande relevância consiste em se avaliar se o balanço radiativo de áreas heterogêneas pode ser obtido com produtos do sensor MODIS (Moderate-Resolution Imaging Spectroradiometer) com qualidade capaz de identificar as diferenças existentes entre áreas agrícolas e de vegetação nativa. Nesse sentido, o presente trabalho objetivou avaliar o desempenho de diferentes metodologias utilizadas na estimativa do saldo de radiação instantâneo, que é a principal fonte de energia a diversos processos naturais que ocorrem à superfície do nosso planeta. Ao mesmo tempo, investiga se as técnicas empregadas proporcionam destacar padrões que diferenciam os ecossistemas em estudo e concluir se a substituição da vegetação nativa por cana-de-açúcar impacta nos valores do albedo e do saldo de radiação, variáveis determinantes nas trocas energéticas entre a atmosfera e a superfície.

\section{MATERIAL E MÉTODOS}

\section{1 Área de estudo e coleta de dados}

A área estudada, representada na Figura 1, localiza-se no estado de São Paulo e abrange o município de Santa Rita

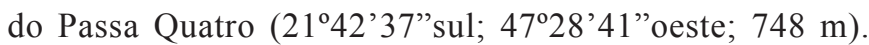
Sua população estimada em 2009 era de 27.557 habitantes e a área municipal era de $754,91 \mathrm{~km}^{2}$, situando-se a $77 \mathrm{~km}$ de Ribeirão Preto. O município pertence à Bacia Hidrográfica do Rio Mogi-Guaçu, que integra a Unidade de Gerenciamento das Bacias Hidrográficas dos Rios Mogi-Guaçu e Pardo e formam, no contexto regional, um dos mais importantes polos socioeconômicos do interior do estado (BRASIL-MME, 2000).

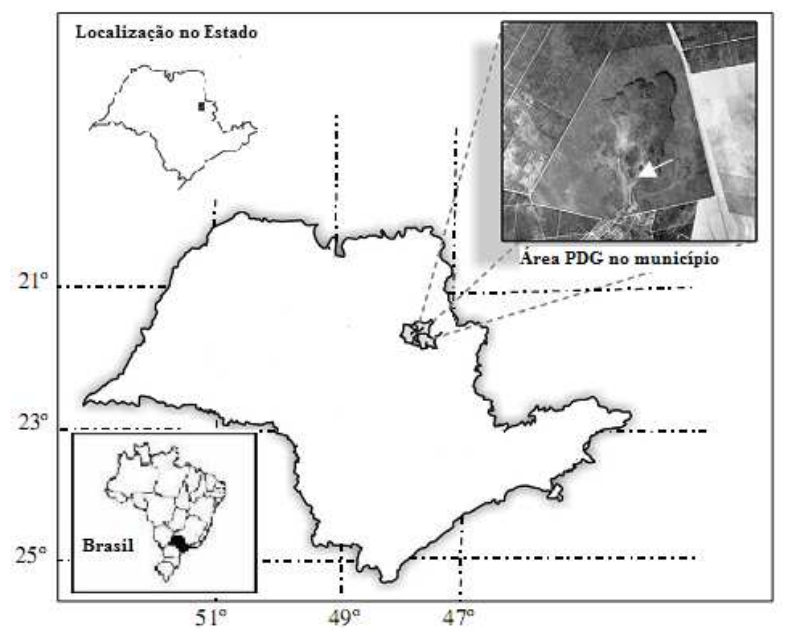

Figura 1: Localização do município de Santa Rita do Passa Quatro no Estado de São Paulo. Em destaque foto aérea onde se observa a Gleba Cerrado Pé-de-Gigante (modificado de Ruggiero et al., 2006). 
O clima da região é normalmente ameno e seco no inverno e quente e úmido no verão (Rocha et al., 2002), com temperatura média anual inferior a $22^{\circ} \mathrm{C}$, pluviosidade anual acima de 1.300 $\mathrm{mm}$ e, durante o verão, temperatura média mensal superior a 22 ${ }^{\circ} \mathrm{C}$ (Pivello et al., 1998).

Os dados de superfície que foram empregados na calibração e validação do Rn, calculado com base nas imagens geradas pelo sensor MODIS, foram obtidos de duas torres micrometeorológicas instaladas em duas áreas do município de Santa Rita do Passa Quatro - SP. Uma delas está localizada na Gleba Cerrado Pé-de-Gigante ( $21^{\circ} 37^{\prime}$ 9,26” S; $47^{\circ} 37^{\prime}$ 56,38" W; $710 \mathrm{~m}$ ) de aproximadamente 1.060 hectares que é uma área de relevante interesse ecológico. A fisionomia predominante na área é de cerrado stricto sensu, com altura do dossel de aproximadamente $10 \mathrm{~m}$, circundado por plantações de eucalipto, citrus e cana-de-açúcar (Rocha et al., 2002). A outra torre foi instalada em uma área de cana-de-açúcar de aproximadamente 351 hectares, na Fazenda São José do Pulador, Usina Santa Rita (2138'13"S; 4747'25"W; 552 m), no município de Luiz Antônio, SP. O talhão foi cultivado com soca de cana-de-açúcar, variedade SP83-2847, com espaçamento entre linhas de 1,5 m e com altura média de $3,5 \mathrm{~m}$ por ocasião das colheitas, realizadas em 14 de abril de 2005 e 10 de maio de 2006 (segundo e terceiro cortes, respectivamente) (Gomes, 2009).

\subsection{Produtos MODIS-Terra}

O sensor MODIS, a bordo dos satélites Terra e Aqua, foi projetado para adquirir dados relativos à interação terra, oceano e atmosfera, possui 36 bandas com cobertura espectral de 0,4 - 14,4 $\mu \mathrm{m}$ e espacial de $250 \mathrm{~m}$ (bandas 1 e 2), $500 \mathrm{~m}$ (bandas 3 a 7) e $1000 \mathrm{~m}$ (bandas 8 a 36), oferecendo cobertura global com tempo de revisita de 1 a dois dias. Os produtos gerados para estudo da terra são produzidos e distribuídos em projeção Sinusoidal, a projeção é dividida em uma grade de quadrados não sobrepostos e adjacentes com cerca de $10 \times 10$ graus (no equador) chamados tiles (fonte: http://modis.gsfc.nasa.gov/).

As imagens MODIS são adquiridas no formato HDF (Hierarchical Data Format) e utilizou-se o software MRT (MODIS Reprojection Tool) para convertê-las no formato GeoTIFF, para posterior processamento com o programa Erdas 8.5. A área de estudo está inserida no tile v11_h13. As imagens selecionadas para a pesquisa são referentes aos dias de ordem do ano 46, 52, 70, 89, 100, 102, 212, 228, 338 e 360, do ano de 2005, adquiridas no endereço: https://wist.echo.nasa.gov/ api/. A Tabela 1 descreve os produtos MODIS utilizados no presente estudo, com os fatores de correção (multiplicativo e ou adicional) que são empregados para converter os valores originais em dados de temperatura $(\mathrm{K})$, emissividade (adimensional), reflectância (adimensional), dentre outros.

\section{i) Albedo da superfície}

Para a sua determinação foram utilizados dois métodos: um proposto por Liang (2000) e outro por Tasumi et al. (2008). O albedo segundo o método Liang (2000) (fundamenta-se na Equação 1:

$$
\begin{aligned}
\alpha_{\mathrm{L}}= & 0,160 \cdot \rho_{1}+0,291 \cdot \rho_{2}+0,243 \cdot \rho_{3}+0,116 \cdot \rho_{4}+0,112 . \rho_{5}+ \\
& 0,081 \rho_{7}-0,0015
\end{aligned}
$$

onde $\rho_{1}(0,62-0,67 \mu \mathrm{m}), \rho_{2}(0,84-0,87 \mu \mathrm{m}), \rho_{3}(0,46-0,48$ $\mu \mathrm{m}), \rho_{4}(0,54-0,56 \mu \mathrm{m}), \rho_{5}(1,23-1,25 \mu \mathrm{m}), \rho_{6}(1,63-1,65 \mu \mathrm{m})$, $\rho_{7}(2,11-2,15 \mu \mathrm{m})$ representam as refletâncias monocromáticas relativas a cada uma das sete bandas espectrais do MODIS, distribuídas por meio do produto MOD09GA.

Para estimativa do albedo conforme a metodologia descrita em Tasumi et al. (2008) ( $\left.\alpha_{T}\right)$, utilizou-se a Equação 2:

\begin{tabular}{|c|c|c|c|c|c|}
\hline Produto & Descrição & $\begin{array}{c}\text { Fator } \\
\text { Multiplicativo }\end{array}$ & $\begin{array}{c}\text { Fator } \\
\text { adicional }\end{array}$ & $\begin{array}{l}\text { Resolução } \\
\text { (espacial e } \\
\text { temporal) }\end{array}$ & Unidades \\
\hline \multirow{5}{*}{ MOD11A1 } & - Temperatura da superfície & 0,02 & - & \multirow{3}{*}{$\begin{array}{c}1 \mathrm{~km} \\
\text { diária }\end{array}$} & Kelvin \\
\hline & - Emissividade das bandas 31 e 32 & 0,002 & 0,490 & & \\
\hline & - Hora da passagem do satélite & 0,1 & & & \multirow{3}{*}{ Hora } \\
\hline & - Refletância da superfície & 0,0001 & - & $0,5 \mathrm{~km}$ & \\
\hline & & & & diária & \\
\hline \multirow[t]{3}{*}{ MOD09GA } & - Ângulo zenital solar & 0,01 & - & & Grau \\
\hline & & & & diária & \\
\hline & - Temperatura do ar & 0,01 & $1,5 \times 10^{4}$ & \multirow{2}{*}{$\begin{array}{l}5 \mathrm{~km} \\
\text { diária }\end{array}$} & Kelvin \\
\hline MOD07_L2 & - Temperatura do ponto do orvalho & 0,01 & $1,5 \times 10^{4}$ & & Kelvin \\
\hline
\end{tabular}

Tabela 1: Descrição dos produtos MODIS utilizados.

Fonte: http://modis.gsfc.nasa.gov/. 


$$
\alpha_{T}=\sum_{b=1}^{n}\left[\rho_{s, b} w_{b}\right]
$$

onde os pesos $w_{\mathrm{b}}$ são os coeficientes de ponderação que representam a fração da radiação solar, que ocorre no intervalo espectral da banda específica, constantes na Tabela 2

\section{ii) Saldo de radiação à superfície ( $(\mathrm{Rn})$}

O Rn foi estimado com base na Equação 3, de três diferentes formas, que diferem quanto ao método de cômputo do albedo da superfície, da radiação de onda curta incidente e da radiação de onda longa incidente. Convencionou-se denominálos de Rn SEBAL (RnS), Rn METRIC (RnM) e RnBisht (RnB), cuja forma geral é dada por:

$$
R n=\operatorname{Rs}(1-\alpha)+R_{L \downarrow}-R_{L \uparrow}-\left(1-\varepsilon_{0}\right) R_{L \downarrow},
$$

em que Rs ( $\mathrm{W} \mathrm{m}^{-2}$ ) é a radiação de onda curta incidente, $\alpha$ (adimensional) é o albedo da superfície; $\mathrm{R}_{\mathrm{L} \downarrow}\left(\mathrm{W} \mathrm{m}^{-2}\right)$ é a radiação de onda longa incidente, proveniente da atmosfera; $\mathrm{R}_{\mathrm{L} \uparrow}\left(\mathrm{W} \mathrm{m}^{-2}\right)$ é a radiação de onda longa emitida pela superfície representada pelo pixel; e $\left(1-\varepsilon_{0}\right) \mathrm{R}_{\mathrm{L} \downarrow}\left(\mathrm{W} \mathrm{m}^{-2}\right)$ representa a radiação de onda longa refletida pela superfície e $\varepsilon_{0}$ é a emissividade da superfície. $\mathrm{O}$ termo $\alpha \mathrm{Rs}\left(\mathrm{W} \mathrm{m}^{-2}\right)$ representa o fluxo de radiação de onda curta refletido pela superfície. A seguir são apresentadas as metodologias empregadas em cada um dos métodos de cômputo do Rn.

A radiação de onda curta incidente $\left(\mathrm{R}_{\mathrm{S} \downarrow}\right)$, para condição de céu claro, foi obtida, para os métodos SEBAL e METRIC, segundo Allen et al. (2007), por:

$$
R_{S \downarrow}=\frac{S_{0} \cdot \cos \theta_{z} \cdot \tau_{s w}}{d^{2}}
$$

onde $\mathrm{S}_{0}$ é a constante solar $\left(1367 \mathrm{~W} \mathrm{~m}^{-2}\right)$; é ângulo zenital solar, em graus, obtido do produto MOD09GA; $\mathrm{d}^{2}=$ quadrado da distância relativa Terra-Sol, obtido segundo Duffie e Beckman (1991):

$$
d^{2}=\frac{1}{1+0,033 \cos (D S A 2 \pi / 365)}
$$

onde DSA é o dia sequencial do ano. A transmissividade atmosférica $\left(t_{s w}\right)$ é o único parâmetro que diferencia os dois métodos. No SEBAL, $\left(t_{\mathrm{sw}}\right)$ foi estimada de acordo com Allen et al. (1998):

$$
\tau_{s w}=0,75+2 \cdot 10^{-5} z
$$

onde $\mathrm{z}$ representa a altitude de cada pixel da imagem, que foi obtido do modelo de elevação digital (DEM) gerado pelo SRTM, junto ao site http://srtm.csi.cgiar.org. Já no método METRIC, $\left(t_{\mathrm{sw}}\right)$ foi determinada conforme Allen et al. (2007) por:

$$
\tau_{s w}=0,35+0,627 \exp \left[\frac{-0,00146 P}{K_{t} \cos \theta_{z}}-0,075\left(\frac{W}{\cos \theta_{z}}\right)^{0,4}\right]
$$

em que $\theta_{z}$ é o ângulo zenital solar, obtido do produto MOD09GA;P é a pressão atmosférica média em $k P a$; $k_{t}$ é o coeficiente de turbidez da atmosfera, sendo $\mathrm{k}_{\mathrm{t}}=1$ para céu claro e $\mathrm{k}_{\mathrm{t}}=0,5$ para turbidez extrema (Allen, 1996); $W$ é a água precipitável (mm), calculada de acordo com equação proposta por Garrison e Adler (1990):

$$
W=0,14 e_{a} P_{a i r}+2.1
$$

A pressão atmosférica pode ser obtida conforme (ASCEEWRI, 2005):

$$
P=101,3\left(\frac{T_{a}-0,0065 z}{T_{a}}\right)^{5,26}
$$

onde $T_{a}(\mathrm{~K})$ é a temperatura do ar e z (m) é a altitude, representada pelo DEM.

Para o método BISHT, na estimativa da radiação de onda curta, foi utilizado esquema de parametrização desenvolvido por Zillman (1972), qual seja:

$$
R_{S \downarrow B}=\frac{S_{0} \cos ^{2} \theta_{z}}{1,085 \cos \theta_{z}+e_{0}\left(2,7+\cos \theta_{z}\right) \times 10^{-3}+\beta}
$$

onde $\theta_{\mathrm{z}}$ ângulo zenital solar, obtido a partir de produto MOD09GA; $\mathrm{S}_{0}$ é a constante solar $\left(1367 \mathrm{~W} \mathrm{~m}^{-2}\right) ; e_{0}$ é a pressão parcial de vapor (hPa), obtida pela equação de ClausiusClapeyron:

$$
e_{0}=6,11 \exp \left[\frac{L_{V}}{R_{V}}\left(\frac{1}{273,15}-\frac{1}{T_{d}}\right)\right]
$$

em que Lv é o calor latente de vaporização da água $\left(2,5 \times 10^{6}\right.$ $\left.\mathrm{J} \mathrm{kg}^{-1} \mathrm{~K}^{-1}\right), R_{v}$ é a constante de vapor de água, $461,50 \mathrm{~kg}^{-1} \mathrm{~K}^{-1}$ (Vianello e Alves, 1987); $T_{d}$ é a temperatura do ar referente ao ponto de orvalho obtida do produto MOD07_L2 (extraída da camada101, que corresponde ao nível de $920 \mathrm{hPa}$ ); $\beta$ é um coeficiente que pode ser utilizado para ajustar os resultados, geralmente fixado em 0,1. Bisht e Bras (2010), Niemelä et al. (2001) e Bisht et al. (2005) mostraram que o valor de $\beta$ adotado por Zillman (1972) tende a superestimar a radiação de ondas curtas incidente, propondo a adoção de $\beta=0,2$, que foi o empregado neste trabalho.

Tabela 2: Coeficientes de ponderação (w_b) da Equação (2), para uso em imagens MODIS (Tasumi et al., 2008)

\begin{tabular}{c|c|c|c|c|c|c|c}
\hline Coeficiente & Banda 1 & Banda 2 & Banda 3 & Banda 4 & Banda 5 & Banda 6 & Banda 7 \\
\hline$W_{b}$ & 0,215 & 0,215 & 0,242 & 0,129 & 0,101 & 0,062 & 0,036 \\
\hline
\end{tabular}


A radiação de onda longa incidente, emitida pela atmosfera na direção da superfície $\left(\mathrm{R}_{\mathrm{L} \downarrow}\right)\left(\mathrm{W} \mathrm{\textrm {m } ^ { - 2 }}\right)$, foi obtida através da equação de Stefan-Boltzmann:

$$
R_{L \downarrow}=\varepsilon_{a} \sigma T_{a}^{4},
$$

em que $T_{a}$ é a temperatura do ar $(\mathrm{K})$ próximo à superfície, $\sigma$ é a constante de Stefan-Boltzmann $\left(\sigma=5,67 \times 10^{-8} \mathrm{~W} \mathrm{~m}^{-2} \mathrm{~K}^{-4}\right)$ e $\varepsilon_{\mathrm{a}}$ é a emissividade atmosférica, calculada segundo modelo proposto por Bastiaanssen et al. (1998), qual seja:

$$
\varepsilon_{a}=a\left(-\ln \tau_{S W}\right)^{b},
$$

onde $a$ e $b$ são coeficientes de calibração cujos valores, de acordo com Bastiaanssen et al. (1998), são respectivamente iguais a 1,08 e 0,265 , quando utilizado o método SEBAL, e 0,85 e 0,09 , conforme Allen et al. (2002), quando utilizado o método METRIC.

Para a metodologia BISHT, a temperatura do ar $\left(\mathrm{T}_{\mathrm{a}}\right)(\mathrm{K})$ na Equação 12 foi obtida do produto MOD07_L2 (extraída da camada 81) e a emissividade atmosférica é estimada de acordo com a Equação 14, proposta por Prata (1996):

$$
\varepsilon_{a}=\left[1-(1+\xi) \exp \left\{-(1,2+3 \xi)^{1 / 2}\right\}\right]
$$

onde $\xi=46,5 \mathrm{e}_{0} / \mathrm{T}_{\mathrm{a}}$

A radiação de onda longa emitida pela superfície representada pelo pixel $\left(\mathrm{R}_{\mathrm{L} \uparrow}\right)\left(\mathrm{W} \mathrm{m}^{-2}\right)$ foi calculada, para as três metodologias propostas, também segundo a equação de Stefan-Boltzmann:

$$
R_{L \uparrow}=\varepsilon_{0} \sigma T_{S}^{4},
$$

onde $\varepsilon_{0}$ é a emissividade da superfície representada pelo pixel e Ts (K) é a temperatura da superfície, obtidos do produto MOD11A1. A emissividade da superfície foi considerada igual à média aritmética das emissividades na faixa espectral das bandas $31(10,78-11,28 \mu \mathrm{m})$ e $32(11,70-12,27 \mu \mathrm{m})$, conforme proposto por Bisht et al. (2005).

\section{iii) Estimativa do saldo de radiação diário}

\section{a) Modelo senoidal}

O saldo de radiação diário, para dias de céu claro, foi estimado a partir de uma modificação do modelo senoidal desenvolvido por Lagouarde e Brunet (1983) e modificado por Bisht et al. (2005), qual seja:

$$
R_{N}(t)=R_{N M A X} \cdot \sin \left[\left(\frac{t_{\text {pass }}-t_{\text {nas }}}{t_{\text {por }}-t_{\text {nas }}}\right) \pi\right]
$$

onde $\mathrm{R}_{\mathrm{NMAX}}$ representa o saldo de radiação máximo observado durante o dia; $t_{\text {pass }}$ representa o instante da passagem do satélite; $t_{\text {nas }}$ e $t_{\text {por }}$ correspondem a hora local em que o saldo de radiação passa a ser positivo e negativo, respectivamente, e estão relacionados com a hora local do nascer e por do Sol.

O saldo de radiação máximo $\left(\mathrm{R}_{\mathrm{nmax}}\right)$ é dado por:

$$
R_{\text {nmax }}=\frac{\mathrm{Rni}}{\sin \left[\left(\frac{t_{\text {PASS }}-t_{\text {nas }}}{t_{\text {por }}-t_{\text {nas }}}\right) \pi\right]}
$$

onde Rni é o saldo de radiação no instante da passagem do satélite.

Foram computados os horários locais do nascer e do por do Sol e comparados, respectivamente, com os instantes medidos em que o saldo de radiação passa a ser positivo e negativo. Visou-se, assim, propor uma adaptação das Equações 16 e 17 às características da área de estudo.

\section{b) Modelo clássico}

O saldo de radiação diário $\left(\mathrm{Rn}_{24 \mathrm{~h}}\right)$, de acordo com De Bruin (1987), pode ser calculado através da Equação 18:

$$
R n_{24 h}=(1-\alpha) R_{S \downarrow 24 h}-a \tau_{s w 24 h}
$$

onde $\alpha$ é o albedo, $\mathrm{R}_{\mathrm{S} \downarrow 24 \mathrm{~h}}\left(\mathrm{~W} \mathrm{~m}^{-2}\right)$ é a radiação solar incidente média diária e $a$ é um coeficiente de regressão entre o saldo de radiação de onda longa diário e a transmissividade atmosférica diária. $\mathrm{O}$ valor de $a$ foi obtido por meio de calibração do modelo acima com base nos dados observados na área em estudo. A transmissividade atmosférica média diária $\left(\tau_{\mathrm{sw} 24 \mathrm{~h}}\right)$ foi obtida através de:

$$
\tau_{S w 24 h}=\frac{R_{S \downarrow_{24 h}}}{R_{S \downarrow T O A_{24 h}}}
$$

onde $\mathrm{R}_{\mathrm{S} \downarrow 24 \mathrm{~h}}\left(\mathrm{~W} \mathrm{~m}^{-2}\right)$ é a radiação solar diária incidente a superfície, que pode ser obtida de estação meteorológica, $\mathrm{R}_{\mathrm{S} \downarrow T O A}\left(\mathrm{~W} \mathrm{~m}^{-2}\right)$ é a radiação solar diária incidente no topo da atmosfera, calculada segundo procedimentos descritos em Iqbal (1984).

\section{iv) Análises estatísticas}

Para a análise dos resultados utilizou-se o Erro Médio Percentual (EMP), o Erro médio absoluto (EMA), o coeficiente de determinação $\left(\mathrm{R}^{2}\right)$, o coeficiente de correlação (r), raiz do erro quadrático médio (REQM), o índice de Willmott (d) (Willmott, 1981) e o índice de desempenho (c) (Camargo e Sentelhas, 1997), descritos na Tabela 3.

\section{RESULTADOS E DISCUSSÃO}

\subsection{Saldo de radiação instantâneo}

A distribuição temporal do saldo de radiação instantâneo medido e estimado pelas diferentes metodologias propostas está mostrada na Figura 2. Pode-se observar que houve 
superestimativa na maior parte dos dias estudados. A metodologia METRIC na estimativa do saldo de radiação instantâneo apresentou erro relativo e absoluto médios, da ordem de $5 \%$ e $29,5 \mathrm{~W} \mathrm{~m}^{-2}$ observados na área PDG (Figura 2a) e de $8 \%$ e $44 \mathrm{~W} \mathrm{~m}^{-2}$, observados na área USR (Figura $2 \mathrm{~b}$ ). Para a metodologia SEBAL o erro médio percentual e o absoluto foram da ordem de 7\% e $40 \mathrm{~W} \mathrm{~m}^{-2}$ (PDG) (Figura 2a) e 9\% e 51 $\mathrm{W} \mathrm{m}^{-2}$ (USR) (Figura 2b), respectivamente. O método BISHT apresentou erros relativos e absolutos médios em torno de 7,5 $\%$ e $43 \mathrm{~W} \mathrm{~m}^{-2}$ (PDG) (Figura 2a) e $10 \%$ e $53,5 \mathrm{~W} \mathrm{~m}^{-2}$ (USR) (Figura 2b), respectivamente. Ataíde (2006) encontrou diferença absoluta, média, da ordem de 57,15 $\mathrm{W} \mathrm{m}^{-2}$ e Bisht et al. (2005) de $59 \mathrm{~W} \mathrm{~m}^{-2}$ no Rn estimado com relação as medidas de campo.

Os valores de Rn medidos na USR variaram de 398,4 $\mathrm{W} \mathrm{m}{ }^{-2}$ no DSA 212 a $801,4 \mathrm{~W} \mathrm{~m}^{-2}$, registrado no DSA 338. De um modo geral, o Rn no cerrado foi maior que na cana-deaçúcar, exceto no DSA 338. Tal comportamento também pode ser observado a partir da análise da Figura 3, que representa a variação espacial do $\mathrm{Rn}$ instantâneo obtido através da metodologia METRIC, para os DSA 52 (céu claro) e 338 (céu nublado). As imagens apresentam claramente que o Rn é maior para a área PDG com relação a USR. A distribuição espacial do saldo mostra-se bastante diferenciada para todo o período de estudo seguindo a sazonalidade relacionada com a incidência da radiação solar, ao valor do ângulo zenital e declinação do Sol.
Os dias sequencias do ano que apresentaram maiores valores na distribuição espacial do Rn, DSA 46 (15 de fevereiro) e 338 (4 de dezembro), são também os dias sequencias em que foram registrados os maiores valores de radiação de onda curta incidente. O DSA 52, cuja declinação solar é -10,82 apresenta valor de saldo menor que o DAS 338, cuja declinação é $-22,13^{\circ}$, que acarreta em maior incidência de radiação solar. Resultados semelhantes foram encontrados por Gomes (2009) e Giongo (2008), onde os maiores valores de saldo foram observados nas imagens referentes aos dias sequencias 22 de fevereiro e 21 de novembro, corroborando com o presente estudo. Também Mendonça (2007) encontrou resultados similares para a região Norte Fluminense, onde a variação espacial do Rn foi maior para os dias sequencias 36 ( 5 de fevereiro) e 320 ( 16 de novembro).

Também se observou que a temperatura da superfície na área com cana-de-açúcar foi sistematicamente superior à obtida na área de cerrado, com impactos esperados na temperatura do ar. Os dados das torres evidenciam que na área com canade-açúcar a $T_{a}$ foi sempre $1,5-2,0^{\circ} \mathrm{C}$ superior à com cerrado. Utilizando os coeficientes de determinação mostrados na Figura 4, foi obtido o coeficiente de desempenho $(c)$, para as metodologias SEBAL, METRIC e BISHT, para as estimativas do Rn (Tabela 4). Para a área PDG, os valores encontrados foram, respectivamente, 0,66, 0,80 e 0,76 o que as classifica, nessa mesma ordem como mediana, boa e boa. Para a área USR

Tabela 3: Índice de Willmott (d) segundo Willmott et al. (1981) e coeficiente de desempenho (c) segundo Camargo e Sentelhas (1997).

\begin{tabular}{c|c|c|c}
\hline $\mathbf{d}$ & $\mathbf{c}$ & Valor de $\boldsymbol{c}$ & Desempenho \\
\hline & & $>0,90$ & Ótimo \\
& & $0,81-0,90$ & Muito Bom \\
$\boldsymbol{d}=\mathbf{1}-\frac{\sum_{\boldsymbol{i}=\mathbf{1}}^{\boldsymbol{N}}\left(\boldsymbol{X}^{\prime}-\boldsymbol{X}\right)^{\mathbf{2}}}{\sum_{\boldsymbol{i}}^{\boldsymbol{N}}\left(\left|\boldsymbol{X}^{\prime}-\overline{\boldsymbol{X}}\right|+|\boldsymbol{X}-\overline{\boldsymbol{X}}|\right)^{\mathbf{2}}}$ & & $0,71-0,80$ & Bom \\
& & $0,51-0,70$ & Mediano \\
& & $0,41-0,50$ & Sofrível \\
& & $0,31-0,40$ & Ruim \\
& & $\leq 0,30$ & Péssimo \\
\hline
\end{tabular}

onde $X$ corresponde ao valor observado, $X^{\prime}$ ao valor estimado, $\bar{X}$ ao valor médio observado e $\mathrm{N}$ a

quantidade de dados analisados.

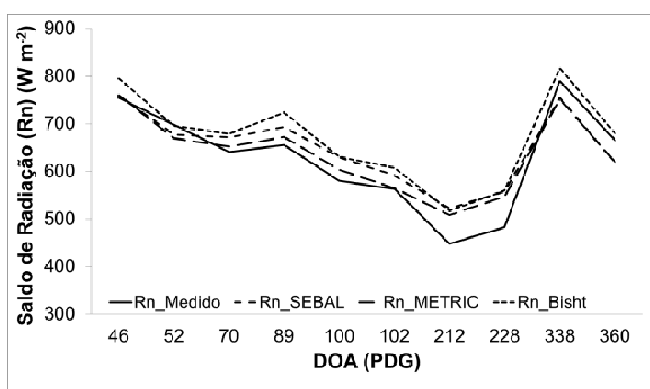

a)

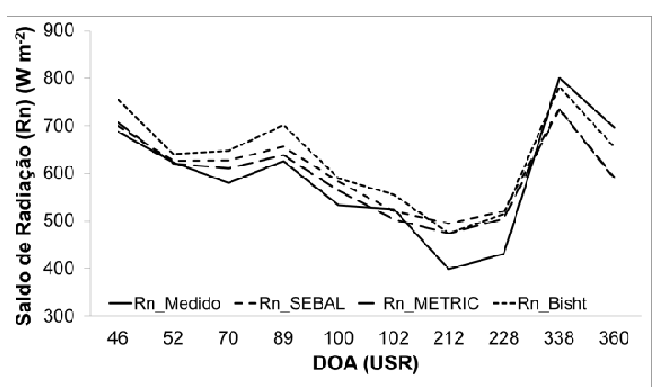

b)

Figura 2: Saldo de radiação medido e estimado através das metodologias SEBAL, METRIC e Bisht para a) Gleba Cerrado Pé-de-Gigante (PDG) a b)Usina Santa Rita (USR). 


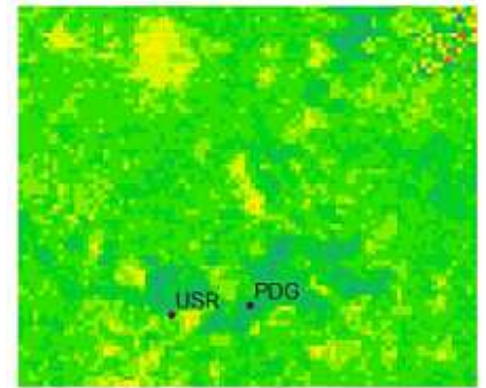

a)

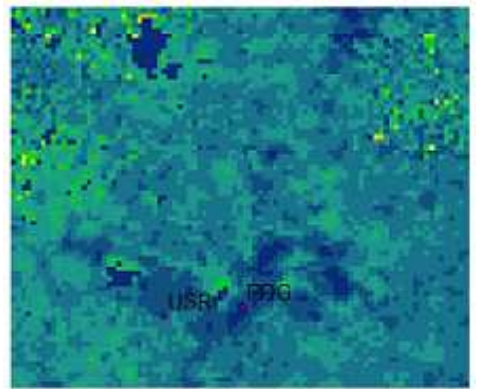

b)

$\begin{array}{llllllllllllllll}400 & 425 & 450 & 475 & 500 & 525 & 550 & 575 & 600 & 625 & 650 & 675 & 700 & 725 & 750 & 775\end{array}$

Figura 3: Distribuição do Saldo de radiação obtido a partir da metodologia METRIC, para o dia 52 a) dia 338 b).

encontraram-se coeficientes de desempenho iguais a 0,59, 0,68 e 0,68 tendo, nesse ecossistema, a classificação de mediana para as três metodologias, de acordo com Camargo e Sentelhas (1997). $\mathrm{O}$ resultado mostra-se interessante pelo fato de evidenciar a necessidade de um tratamento estatístico que não leve em conta apenas os erros médios e absolutos percentuais, pois em alguns casos pode haver uma compensação de erros resultando um valor médio com pequena diferença entre estimativas e observações.

Bisht et al. (2005) encontraram REQM da ordem de $74 \mathrm{~W}$ $\mathrm{m}^{-2}$. No presente estudo, como mostrado na Tabela 4, os valores de REQM variaram de $36,16 \mathrm{~W} \mathrm{~m}^{-2}$ para a metodologia METRIC a $62,47 \mathrm{~W} \mathrm{~m}^{-2}$ para a metodologia SEBAL. Esses resultados evidenciam o melhor desempenho do método METRIC, já que apresentou os maiores valores de $c$ e menores valores de REQM. Apesar de ter apresentado a melhor correlação $(r)$, a metodologia BISHT não superou a METRIC, pois as demais análises estatísticas levam em consideração, também, o erro relativo médio no seu cômputo, sendo que o RnM apresentou os menores erros desse tipo.

\subsection{Saldo de radiação diário}

\subsubsection{Modelo clássico}

Aplicando-se a Equação 18 aos dados observados em superfície, com $a=110$, obteve-se os coeficientes de determinação de 0,93 para as áreas PDG (Figura 5a) e USR (Figura 5b). Efetuou-se a calibração da Equação 18 através dos dados observados no experimento a partir da relação de dispersão entre o saldo diário de radiação de onda longa e a transmissividade diária. Obteve-se, então, para a área de estudo, o coeficiente $a$ da equação clássica, mostrado na Equação 20:

$$
R n_{24 h}=\left(1-\alpha_{24 h}\right) R_{S \downarrow 24 h}-122,83 \tau_{s w 24 h}
$$

Na Figura 6 estão os valores observados e os estimados através da Equação 20 com a respectiva correlação.
Efetuando-se a calibração sem forçar a passagem da reta pela origem, obteve-se uma nova equação, alternativa (Equação 21):

$$
R n_{24 h}=\left(1-\alpha_{24 h}\right) R_{S \downarrow 24 h}-352,95 \tau_{s w 24 h}+159,56
$$

Os resultados mostram uma melhor correlação entre medidas e estimativas na aplicação das Equações 18 e 20 para a área PDG, já para a Equação 21, o melhor desempenho ocorreu na área USR (Figura 7). Os valores de $\mathrm{R}^{2}$, porém, apresentaram pequena variação quando comparados entre si, ou seja, para as três equações e, considerando-se o $\mathrm{R}^{2}$ médio para os dois sítios, encontra-se o melhor resultado para a Equação 21. As calibrações propostas demonstram, então, confiabilidade, já que geram resultados semelhantes à metodologia clássica, a qual já foi aplicada e validada em diversas áreas do globo.

\subsubsection{Modelo Senoidal}

Para a estimativa do $\mathrm{Rn}$ diário, a partir do modelo senoidal, optou-se por escolher apenas o Rn que foi denominado $\mathrm{RnB}$, no intuito de aplicar uma metodologia "autônoma", como proposta por Bisht et al. (2005). Neste caso a estimativa do Rn diário se dá sem necessidade de medida de superfície.

Propõe-se, aqui, uma modificação da equação senoidal, de acordo com Ataíde (2006), baseada em observações feitas durante os dias selecionados. Foram acrescentados 55,08 minutos a hora local do nascer do Sol $(0,918 \mathrm{~h})$ e diminuídos 25,38 minutos $(0,423 \mathrm{~h})$ a hora local do por do Sol. As Equações 16 e 17 tornam-se, então:

$$
\begin{aligned}
R_{N}(t)= & R_{N M A X} \cdot \\
& \sin \left[\left(\frac{t_{\text {pass }}-\left(t_{\text {nas }}+0,918\right)}{\left(t_{\text {por }}-0,423\right)-\left(t_{\text {nas }}+0,918\right)}\right) \pi\right]
\end{aligned}
$$


a)

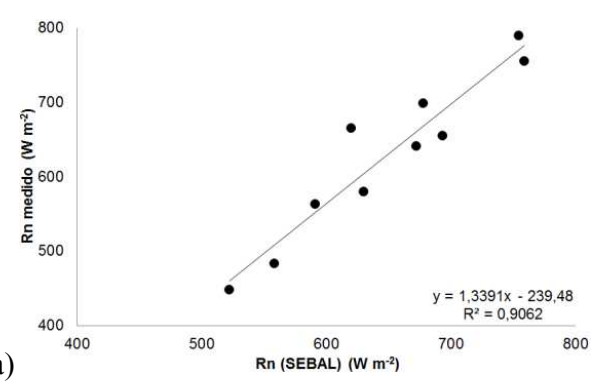

c)

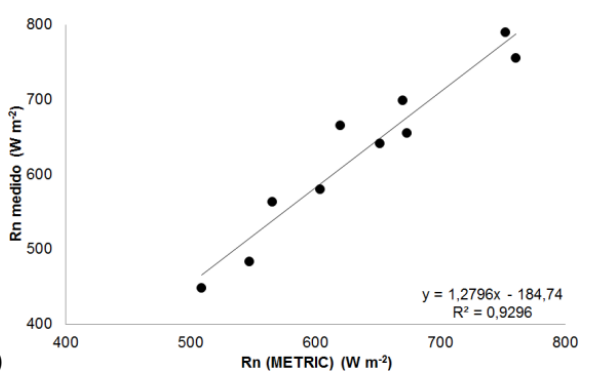

e)

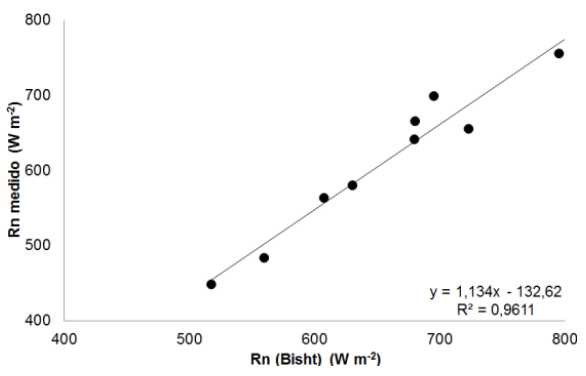

b)

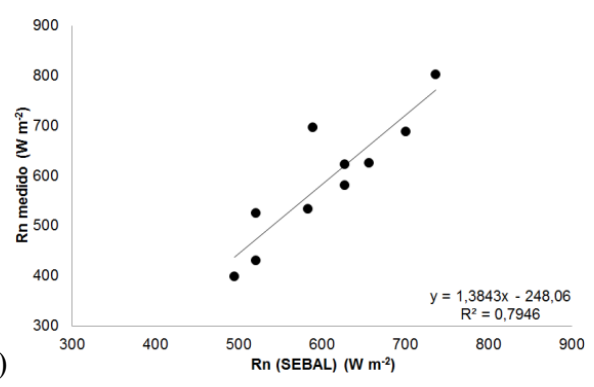

d)
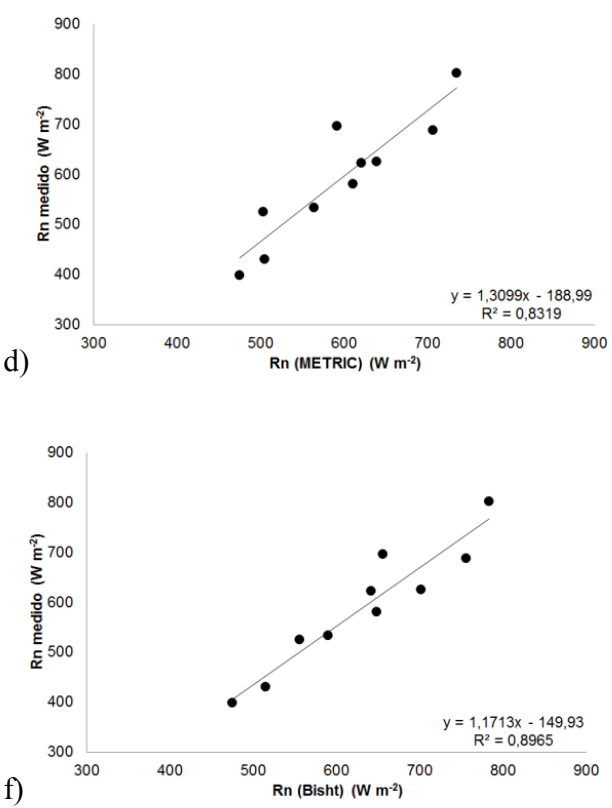

Figura 4: Correlação entre os valores do Rn medidos nas torres micrometeorológicas do Cerrado (a, c, e) e da Usina Santa Rita (b, d, f) e os estimados através das metodologias descritas.

Tabela 4: Correlação (r), Índice de Willmott $(d)$, Coeficiente de desempenho de Camargo e Sentelhas (c) e Raiz do Erro Quadrático Médio (REQM) para as estimativas do Rn através das metodologias BISHT (RnB), METRIC (RnM) e SEBAL (RnS).

\begin{tabular}{ccccccccc}
\hline & \multicolumn{3}{c}{ PDG } & \multicolumn{1}{c}{ USR } \\
\hline & $\boldsymbol{r}$ & $\boldsymbol{d}$ & $\boldsymbol{c}$ & $\boldsymbol{R E} \boldsymbol{Q M}$ & $\boldsymbol{r}$ & $\boldsymbol{d}$ & $\boldsymbol{c}$ & $\boldsymbol{R} \boldsymbol{E} \boldsymbol{M}$ \\
RnS & 0,92 & 0,72 & 0,66 & 45,35 & 0,85 & 0,63 & 0,59 & 62,47 \\
RnM & 0,94 & 0,86 & 0,80 & 36,16 & 0,89 & 0,75 & 0,68 & 54,53 \\
RnB & 0,97 & 0,78 & 0,76 & 48,98 & 0,93 & 0,64 & 0,68 & 58,52 \\
\hline
\end{tabular}

$$
R_{N M A X}=\frac{I N R}{\sin \left[\left(\frac{t_{P A S S}-\left(t_{\text {nas }}+0,918\right)}{\left(t_{\text {por }}-0,423\right)-\left(t_{\text {nas }}+0,918\right)}\right) \pi\right.}
$$

O modelo senoidal original, como descrito através da Equação 16, desconsidera o Rn no período noturno, impondo para o mesmo o valor zero. Araújo (2010) propôs uma equação que prevê que o Rn noturno é aproximadamente $8 \%$ do valor do Rn máximo observado durante o dia. Observando os dados do experimento, para os dias de estudo, chegou-se, similarmente, para o período noturno, à relação:

$$
R n(t)=-0,08245 \cdot R n_{M A \lambda}
$$

As curvas geradas ao aplicar-se o modelo, para as áreas da PDG e USR em dias de céu-claro (DSA 52) e nublado (DSA 338), encontram-se nas Figuras 8 e 9, respectivamente. Para os dias de céu claro percebe-se uma grande aproximação entre estimativas e observações, ocorrendo o melhor ajuste para a área PDG. Já para os dias com nuvens ocorreu uma superestimativa no ciclo diurno gerado a partir do $\mathrm{Rn}(\mathrm{t}) \mathrm{PDG}$ _Bisht para os dois sítios, com relação as medidas de superfície. Esse é o comportamento esperado já que a presença de nuvens ocasiona 
a)

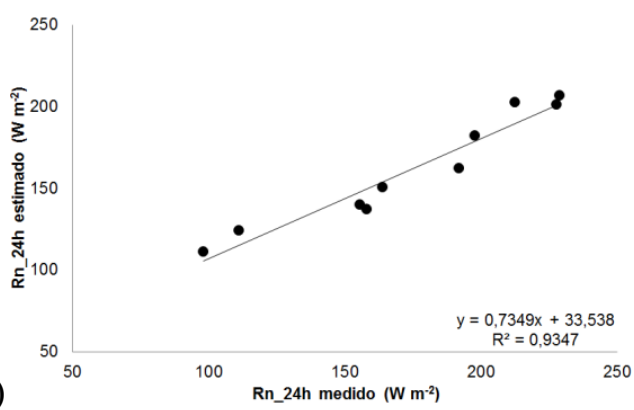

b)

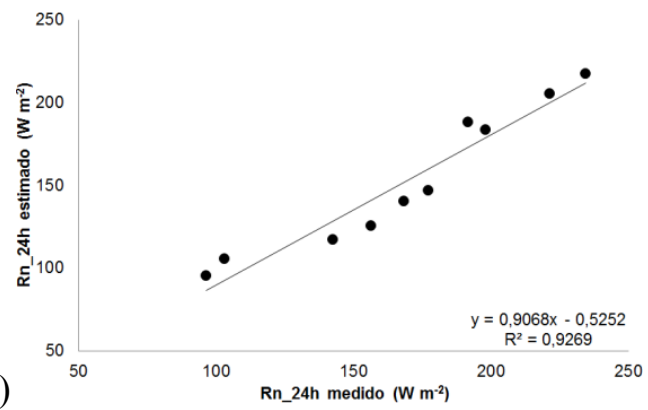

Figura 5: Correlação entre os valores diários do Rn observado e estimado através da equação 18 para a) PDG e b) USR.

a)

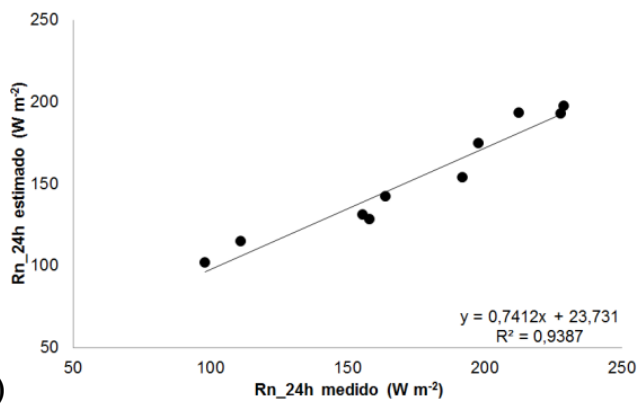

b)

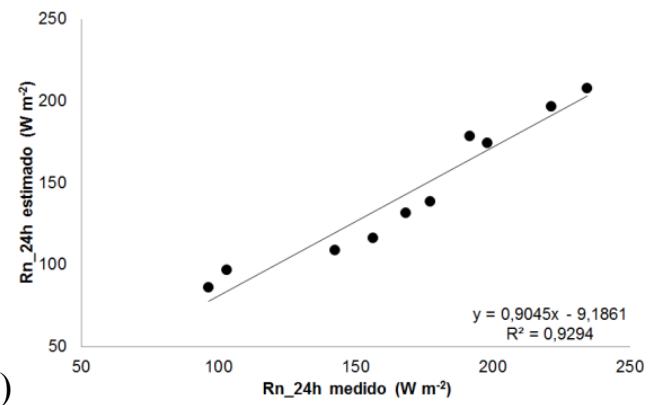

Figura 6: Correlação entre os valores diários do Rn observado e estimado através da equação 20 para a) PDG e b) USR.

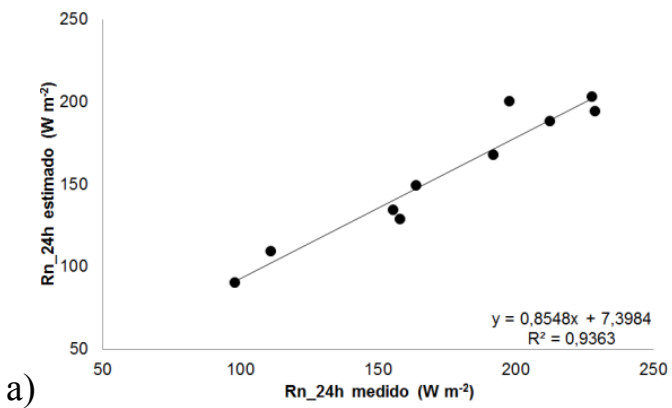

b)

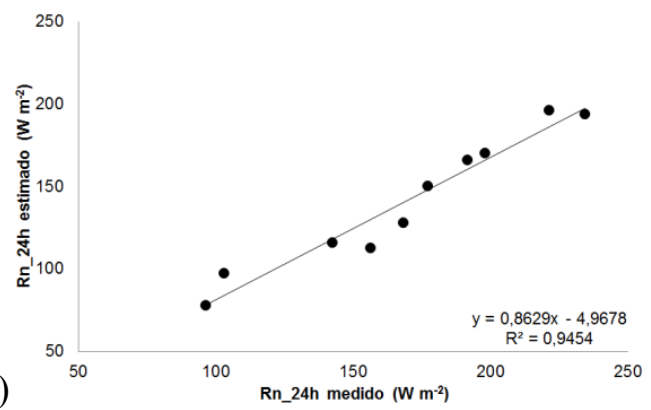

Figura 7: Correlação entre os valores diários do Rn observado e estimado através da Equação 21 para a) PDG e b) USR.

um maior aprisionamento da radiação de onda longa, elevando assim o saldo de radiação. Também é interessante ressaltar que o Rn(t)PDG mostra o ciclo diurno que deveria ser seguido caso se tratasse de um dia de céu claro.

Aplicando-se o modelo senoidal aos dados observados no experimento, desconsiderando o saldo no período noturno (como proposto no modelo original) e depois aplicando a Equação 24 a tal período, observa-se que o erro diminuiu consideravelmente. $\mathrm{O}$ erro relativo médio caiu de $22,8 \%$ para $6,9 \%$ na área de cerrado (PDG) e de $22,9 \%$ para $4,7 \%$ na área de cana-de-açúcar (USR).

As Figuras 10 e 11 apresentam as correlações entre os valores obtidos a partir do modelo senoidal aplicado aos dados de satélite, primeiro com os dados observados no experimento (Figuras 10a e 11a) e depois com os resultados encontrados a partir do modelo senoidal aplicado a esses dados observados (Figuras 10b e 11b). Percebe-se que houve um aumento na correlação de 0,95 para 0,98 (PDG) e de 0,95 para 0,97 (USR). Nos resultados encontrados por Ataíde (2006), o valor de $\mathrm{R}^{2}$ aumentou de 0,93 para 0,99, após a correção de nuvens. Vale acrescentar que ele desconsiderou o saldo de radiação no período noturno, enquanto no presente estudo considerou-se todo o período de 24 horas. Esses resultados comprovam a eficácia na aplicabilidade do modelo para dias de céu claro.

\section{CONCLUSÕES}

Diante dos resultados encontrados pode-se observar que as metodologias empregadas para o cômputo do saldo 

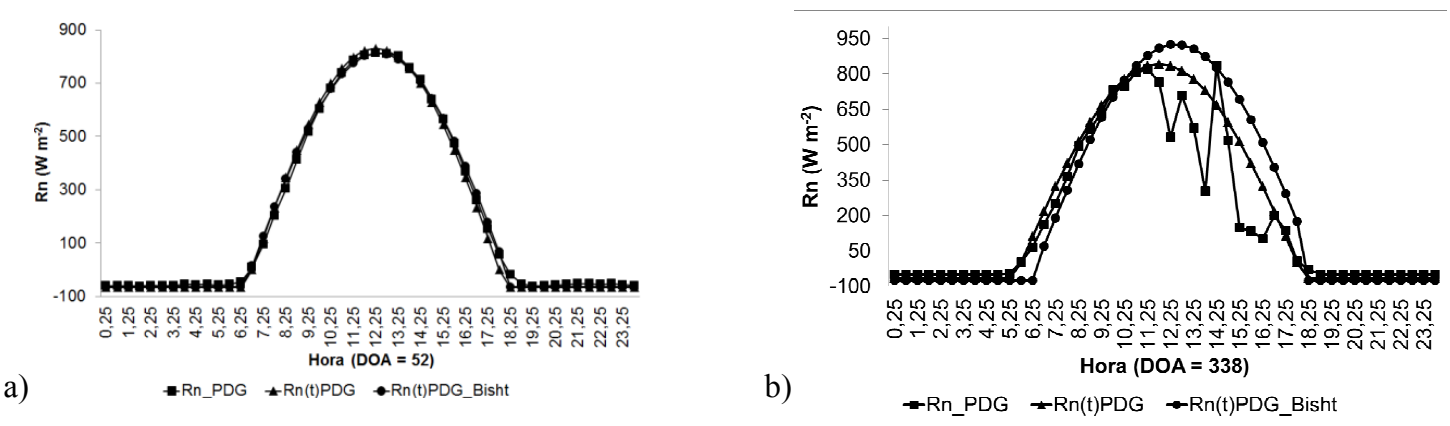

Figura 8: Modelo para estimativa do ciclo diurno do saldo de radiação (W m-2) aplicado aos DSA 52 (a) (céu-claro) e 338 (b) (nublado) para a Gleba Pé-de-Gigante. A linha com marcadores quadrados representam os dados observados no experimento Rn_PDG, a linha com marcadores triangulares representam os valores estimados a partir dos dados observados através das equações 16 e 17 (Rn(t)PDG) e a linha com marcadores redondos representam os valores estimados a partir do Rn obtido através do sensor MODIS, com a metodologia Bisht, utilizando as equações 22, 23 e 24 Rn(t)PDG_Bisht.
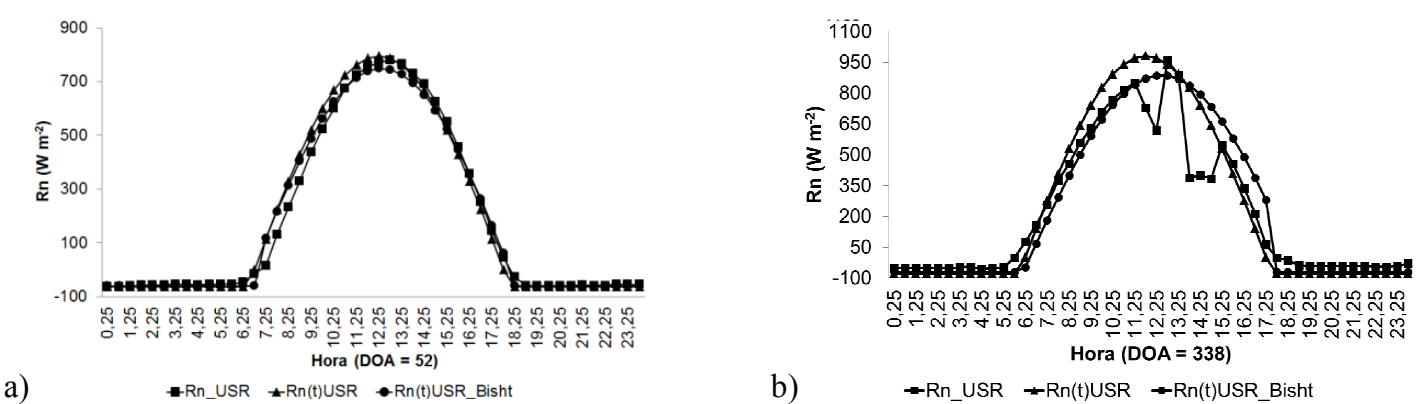

Figura 9: Modelo para estimativa do ciclo diurno do saldo de radiação (W m-2) aplicado aos DSA 52 (a) (céu-claro) e 338 (b) (nublado) para a Usina Santa Rita.

a)

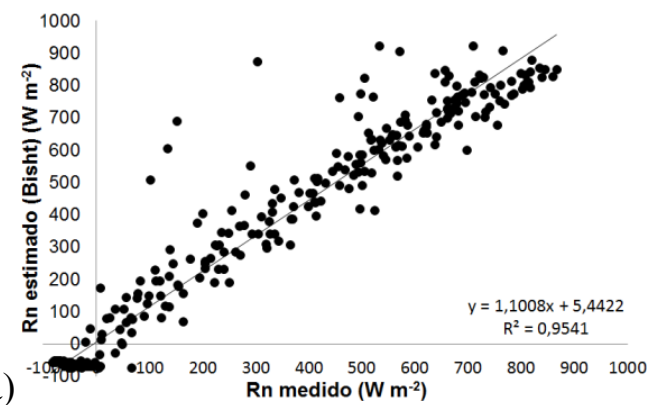

b)

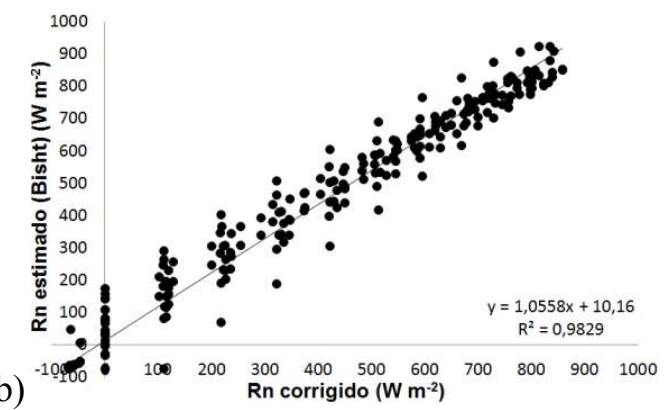

Figura 10: a) Dispersão entre os valores do saldo de radiação estimados (para intervalos de meia hora) e valores medidos e b) dispersão entre os valores do saldo de radiação estimados (para intervalos de meia hora) e valores corrigidos, para a área PDG.

a)

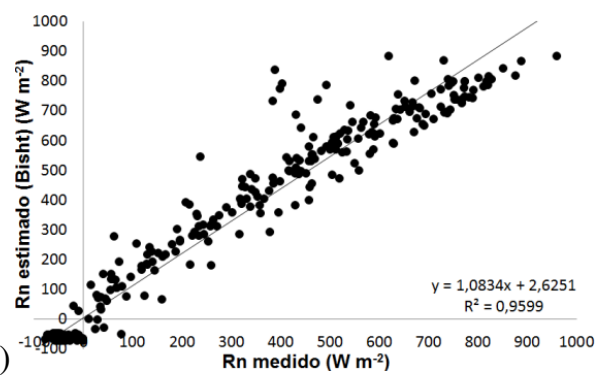

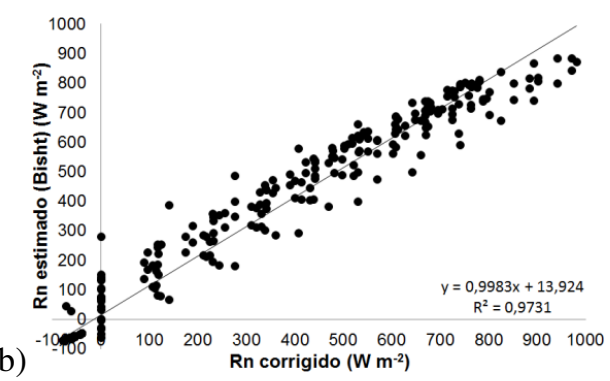

Figura 11: a) Dispersão entre os valores do saldo de radiação estimados (para intervalos de meia hora) e valores medidos e b) dispersão entre os valores do saldo de radiação estimados (para intervalos de meia hora) e valores corrigidos, para a área USR. 
de radiação instantâneo mostraram-se bastante satisfatórias, havendo um melhor desempenho para o METRIC. Dentre as mesmas, o fato de a metodologia BISHT não necessitar de dados de superfície mostra-se útil para as estimativas do Rn.

O modelo senoidal modificado para a estimativa do saldo de radiação diário, apresentou um alto grau de operacionalidade, conseguindo estimar o ciclo diurno do Rn a partir de dados de satélite, sendo o mesmo, indicado para dias sem presença de nuvens.

A utilização das metodologias empregadas, para condições de céu claro, na detecção de alterações microclimáticas decorrentes da mudança no uso do solo, foi satisfatória, visto que os resultados do albedo e $\mathrm{Rn}$ foram nitidamente diferenciados para cada ecossistema em estudo. O Rn no cerrado mostrou-se sempre maior do que o da cana-de-açúcar, indicando que, nessas áreas nativas, há, portanto, uma maior quantidade de energia a ser convertida em calor sensível e latente.

\section{AGRADECIMENTOS}

Os autores agradecem a Coordenação de Aperfeiçoamento de Pessoal de Nível Superior (CAPES) pela concessão de bolsas de Mestrado e Doutorado à primeira autora e ao Conselho Nacional de Desenvolvimento Científico e Tecnológico (CNPq) pela concessão da bolsa de Produtividade em Pesquisa ao segundo e terceiro autores. Similarmente, agradecem ao Prof. Dr. Humberto Ribeiro Rocha pela disponibilização dos dados medidos à superfície para fins de validação das metodologias empregadas.

\section{REFERÊNCIAS BIBLIOGRÁFICAS}

ALLEN, R. G. Assessing integrity of weather data for use in reference evapotranspiration estimation. Journal of Irrigation and Drainage Engineering, v.122, p.97-106, 1996.

ALLEN, R. G.; PEREIRA, L. S.; RAES, D.; SMITH, M. Crop evapotranspiration: Guidelines for computing crop water requirements. United Nations FAO, Irrigation and Drainage, N.Y., Paper No. 56, 1998.

ALLEN, R. G.; TASUMI, M.; TREZZA, R. Satellite-based energy balance for mapping evapotranspiration with internalized calibration (METRIC) - Model. Journal of Irrigation and Drainage Engineering, v.133, n.4, p.380394, 2007.

ALLEN, R. G.; TREZZA, R.; TASUMI M. Surface energy balance algorithms for land. Advance training and user's manual, version 1.0, p.98, 2002.

ARAÚJO, A. L. Calibração do balanço de radiação diário através de dados de superfície e de sensores orbitais.
Dissertação (Mestrado em Meteorologia). Universidade Federal de Campina Grande, Campina Grande - PB, 115p, 2010.

ASCE-EWRI. The ASCE standardized reference evapotranspiration equation. ASCE-EWRIStandardization of Reference Evapotranspiration Task Committee Rep., ASCE, 2005.

ATAIIDE, K. R. P. Determinação do saldo de radiação e radiação solar global com produtos do sensor MODIS Terra e Aqua. Dissertação (Mestrado em Meteorologia) Universidade Federal de Campina Grande, Campina Grande - PB, 97p, 2006.

BASTIAANSSEN, W. G. M.; MENENTI, M.; FEDDES, R. A.; HOLTSLAG, A. A. M.A remote sensing surface energy balance algorithm for land (SEBAL). 1: Formulation. Journal of Hydrology, v.212-213, p.198-212, 1998.

BISHT, G.; BRAS, R. L. Estimation of net radiation from the MODIS data under all sky conditions: Southern Great Plains case study. Remote Sensing of Environment, v. 114, n.7, p. 1522-1534, 2010.

BISHT, G.; VENTURINI, V.; ISLAM, S.; JIANG, L. Estimation of the net radiation using MODIS (Moderate Resolution Imaging Spectroradiometer) data for clear-sky days. Remote Sensing of Environment, v.97, n.1, p.52-67, 2005.

BRASIL. Ministério de Mina e Energia. Secretaria de Minas e Metalurgia. Serviço Geológico do Brasil. Superintendência Regional de São Paulo. Atlas Ambiental das Bacias dos Rios Mogi-Guaçu e Pardo, SP: subsídios para o planejamento territorial e gestão ambiental. Coordenadoria de Planejamento Ambiental, 77p, 2000.

CAMARGO, A. P.; SEntelhas, P.C. Avaliação do desempenho de diferentes métodos de estimativa da evapotranspiração potencial no estado de São Paulo, Brasil. Revista Brasileira de Agrometeorologia, v.5, p.89-97, 1997.

DE BRUIN, H. A. R. From Penman to Makkink. in: HoogharT, J. C. (Ed.), Proceedings and information: TNO Commitee on Hydrological. Gravennhage, The Netherlands, v.39, p.5-31, 1987.

DUFFIE, J. A.; BECKMAN, W. A. Solar engineering of thermal process, $2^{\text {nd }}$ Ed., Wiley, N. Y. 1991.

GARRISON, J. D.; ADLER, G. P. Estimation of precipitable water over the United States for application to the division of solar radiation into its direct and diffuse components. Solar Energy, v.44, p.225-241, 1990.

GIONGO, P. R. Estimativa do balanço de radiação com técnicas de Sensoriamento remoto e dados de superfície. Dissertação (Mestrado em Engenharia Agrícola). Universidade Federal Rural de Pernambuco, Recife-PE, 94p., 2008. 
GOMES, H. F. Balanços de radiação e energia em áreas de cultivo de cana-de-açúcar e cerrado no estado de São Paulo mediante imagens orbitais, 119p. Tese (Doutorado em Meteorologia). Universidade Federal de Campina Grande, Campina Grande - PB, 2009.

IQBAL, M. An introduction to solar radiation. Library of Congress Cataloging in Publication Data: Academic Press Canada, 408p, 1984.

LAGOUARDE, J. P. E BRUNET, Y. A simple model for estimating the daily upward longwave surface radiation flux from NOAA-AVHRR data. International Journal of Remote Sensing, v.14, n.5, p.907-925, 1983.

LIANG, S. Narrowband to broadband conversions of land surface albedo I Algorithms. Remote Sensing of Environment, v.76, p. 213- 238, 2000.

MENDONÇA, J. C. Estimativa da evapotranspiração regional utilizando imagens digitais orbitais na região Norte Fluminense. Tese (Doutorado em Produção Vegetal), Universidade Estadual do Norte Fluminense Darcy Ribeiro, Rio de Janeiro, 145p, 2007.

NEGRÓN-JUAREZ, R. I. Variabilidade Climática Regional e Controle da Vegetação no Sudeste: Um estudo de Observações sobre cerrado e Cana-de-açúcar e Modelagem Numérica da Atmosfera. Tese (Doutoramento em Ciências Atmosféricas) Instituto de Astronomia e Geofísica. Universidade de São Paulo. 159 p., 2004.

NIEMELÄ, S.; RÄISÄNEN, P.; SAVIJÄRVI, H. Comparison of surface radiative flux parameterizations: Part II. Short wave radiation. Atmospheric Research, v.58, p.141-154, 2001.

PIVELLO, V.R.; BITENCOURT, M.D.; MANTOVANI, W.; MESQUITA JR. H.N.; BATALHA, M.A.; SHIDA, C. Proposta de zoneamento ecológico para a reserva de cerrado Pé-de-Gigante (Santa Rita do Passa Quatro, SP). Revista Brasileira de Ecologia, v.2, p.108-118, 1998.
PRATA, A. J. A new long-wave formula for estimating downward clear-sky radiation at the surface. Quarterly Journal of the Royal Meteorological Society, v.122, 1127-1151, 1996.

ROCHA, H. R; FREITAS, H. C.; ROSOLEM, R.; NEGRÓNJUÁREZ, R. I.; TANNUS, R.; LIGOMA, CABRAL O. M.; DIAS, M. A. F. Measurements of $\mathrm{CO}_{2}$ exchange over a woodland savanna (Cerrado sensu stricto) in southeast Brazil. Biota Neotropica, v.2, n.1, p.1-11, 2002.

SECRETARIA DE ESTADO MEIO AMBIENTE DO ESTADO DE SÃO PAULO - SMA, 2010: Relatório de Qualidade Ambiental do Estado de São Paulo. Disponível em http:// www.ambiente.sp.gov.br.

TASUMI, M.; ALLEN, R. G.; TREZZA, R. At-surface reflectance and albedo from satellite for operational calculation of land surface energy balance. Journal of Hydrologic Engineering, v.13, p.51-63, 2008.

TATSCH J. D. Uma análise dos fluxos de superfície e do microclima sobre cerrado, cana-de-açúcar e eucalipto, com implicações para mudanças climáticas regionais. Dissertação (Mestrado em Ciências). Universidade de São Paulo, São Paulo - SP, 132p, 2006.

VIANELlO, R. L.; ALVES, A. R. Meteorologia básica e aplicação. Universidade Federal de Viçosa, p.449, 1987.

WILlMOTT, C.J. On the validation of models. Physical Geography, v.2, p.184-194, 1981.

ZILLMAN, J. W. A study of some aspects of the radiation and heat budgets of the southern hemisphere oceans. Meteorology Study 26, Bureau of Meteorology, Australia Dept. of the Inter, 1972. 